Supporting Information

\title{
Ultrathin Reduced Graphene Oxide/Organosilica Hybrid Membrane for Gas Separation
}

Yayun Zhao, Chen Zhou, Chunlong Kong and Liang Chen*

Ningbo Institute of Materials Technology and Engineering, Chinese Academy of Sciences, Ningbo, Zhejiang 315201, P. R. China

University of Chinese Academy of Sciences, Beijing 100019, P. R. China

*E-mail: chenliang@nimte.ac.cn 


\section{Contents}

Figure S1. AFM measurements of GO nanosheets.

Figure S2. (a) Digital photographs and (b) Zeta potential of organosilica polymer sol and GO dispersed in organosilica polymer sol (GO/organosilica =1/1 wt./wt.); (c) Photographs of the blank hollow fiber (below) and the GO-Si1/1 membrane (above).

Figure S3. SEM images of the surface and cross-section view of $(A, B)$ pure organosilica membrane, $(C$, D) GO-Si1/2, (E, F) GO-Si1/1.5, (G, H) GO-Si1.5/1 and (I, J) GO-Si2/1 membranes.

Figure S4. XRD patterns of pristine organosilica, various $\mathrm{r}-\mathrm{GO} /$ organosilica membranes.

Figure S5. SAXS curves for organosilica and r-GO/organosilica, in which the X-ray beam was perpendicular to the film plane. The slope at low $q$-range (Guinier region) were used to determine fractal dimensions.

Figure S6. FT-IR spectra of GO, organosilica and r-GO/organosilica membranes, (a) from 4000 to 400 $\mathrm{cm}^{-1}$, (b) from 1450 to $1250 \mathrm{~cm}^{-1}$.

Figure S7. Evolution of C1s XPS spectrum of GO, Si 2p XPS spectrum of organosilica, and C1s and Si $2 \mathrm{p}$ XPS spectra of r-GO/organosilica membranes.

Figure S8. (a) Gas permeance and (b) the ideal selectivities for the corresponding gas pairs of the BTESEderived organosilica membrane, GO-Si1/2, GO-Si1/1.5, GO-Si1/1, GO-Si1.5/1 and GO-Si2/1 membranes. Permeances were measured at $\Delta \mathrm{p}=0.2 \mathrm{MPa}$ and $\mathrm{T}=25^{\circ} \mathrm{C}$.

Figure S9. Schematic process of gas molecules permeating through the r-GO/organosilica membrane.

Figure S10. The ideal selectivities for $\mathrm{H}_{2} / \mathrm{C}_{3} \mathrm{H}_{8}, \mathrm{H}_{2} / i-\mathrm{C}_{4} \mathrm{H}_{10}, \mathrm{CO}_{2} / \mathrm{C}_{3} \mathrm{H}_{8}$ and $\mathrm{CO}_{2} / i-\mathrm{C}_{4} \mathrm{H}_{10}$ of GO-Si1/1 hybrid membranes (insert: single gas permeances of $\mathrm{H}_{2}, \mathrm{CO}_{2}, \mathrm{C}_{3} \mathrm{H}_{8}$ and $i-\mathrm{C}_{4} \mathrm{H}_{10}$ ). Permeance was measured at $\Delta \mathrm{p}=0.2 \mathrm{MPa}$ and $\mathrm{T}=25^{\circ} \mathrm{C}$.

Figure S11. SEM images of the surface and cross-section view of r-GO/organosilica membranes fabricated by (A, B) 2-fold diluted coating solution and (C, D) 4-fold diluted coating solution.

Figure S12. (a) Single gas permeances through r-GO/organosilica membranes fabricated by original, 2 fold diluted and 4-fold diluted coating solution; (b-c) the ideal selectivities for the corresponding gas pairs. Permeances were measured at $\Delta \mathrm{p}=0.2 \mathrm{MPa}$ and $\mathrm{T}=25^{\circ} \mathrm{C}$.

Figure S13. Pure-component $\mathrm{CO}_{2}$ and $\mathrm{CH}_{4}$ adsorptions of $\mathrm{GO} /$ organosilica powders at $25^{\circ} \mathrm{C}$.

Figure S14. $\mathrm{CO}_{2}$ and $\mathrm{CH}_{4}$ adsorptions of $\mathrm{r}-\mathrm{GO}$ /organosilica powders at $25^{\circ} \mathrm{C}$ between 0.01 bar to 0.2 bar. Figure S15. Sorption isotherms of neat organosilica powders (a) and r-GO organosilica powders (b) for $\mathrm{CO}_{2}$ and $\mathrm{CH}_{4}$ at $273 \mathrm{~K}$ and $298 \mathrm{~K}$.

Figure S16. Thermal stability measurement of the $\mathrm{r}-\mathrm{GO} /$ organosilica membrane for the separation of an equimolar $\mathrm{CO}_{2} / \mathrm{CH}_{4}$ mixture at $25^{\circ} \mathrm{C}$.

Figure S17. Thermal stability measurement of the $\mathrm{r}-\mathrm{GO} /$ organosilica membrane for the separation of an equimolar $\mathrm{CO}_{2} / \mathrm{CH}_{4}$ mixture under wet state at $150{ }^{\circ} \mathrm{C}$.

Figure S18. (a) The gas fluxes of $\mathrm{CO}_{2}$ and $\mathrm{CH}_{4}$ from an equimolar $\mathrm{CO}_{2} / \mathrm{CH}_{4}$ mixture, and (b) their gas permeances and $\mathrm{CO}_{2} / \mathrm{CH}_{4}$ selectivity of the $\mathrm{r}-\mathrm{GO} /$ organosilica membrane as function of the $\mathrm{CO}_{2}$ feed pressure at $25^{\circ} \mathrm{C}$.

Table S1. Single gas permeances of $\mathrm{H}_{2}, \mathrm{CO}_{2}$ and $\mathrm{CH}_{4}$ and the ideal selectivities of $\mathrm{H}_{2} / \mathrm{CH}_{4}$ and $\mathrm{CO}_{2} / \mathrm{CH}_{4}$ at room temperature and $0.2 \mathrm{MPa}$ of 3 tested hybrid membranes $(\mathrm{r}-\mathrm{GO} /$ organosilica $=1 / 1)$ prepared with the same procedures.

Table S2. Comparison of gas separation performance of r-GO/organosilica membrane with other reported $\mathrm{H}_{2}$-selective $\mathrm{GO}$ membranes at room temperature.

Table S3. Comparison of gas separation performance of $\mathrm{r}-\mathrm{GO} /$ organosilica membrane with other reported $\mathrm{CO}_{2}$-selective membranes. 

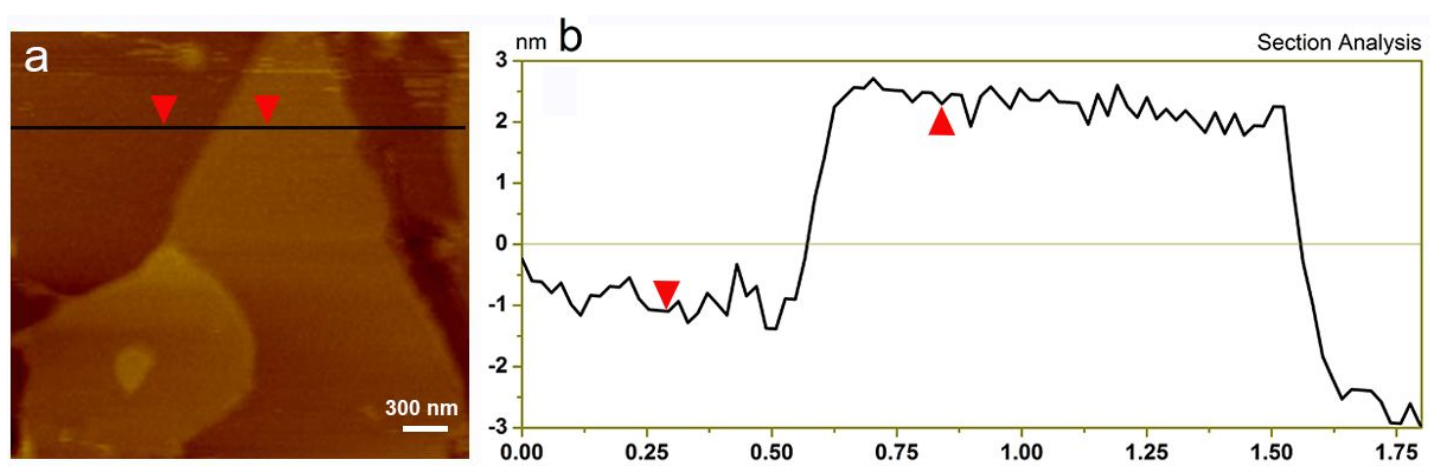

Figure S1. AFM measurements of GO nanosheets.
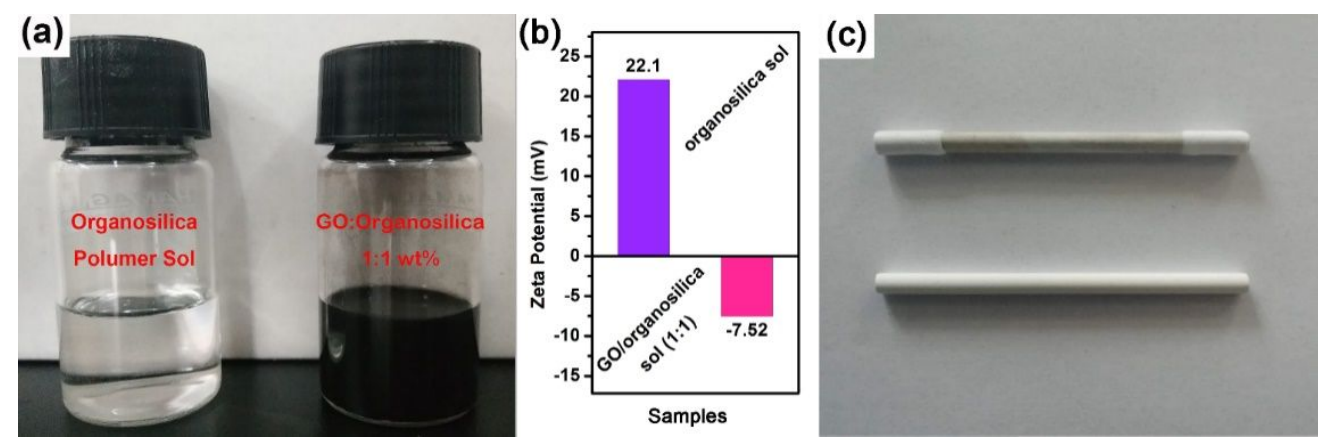

Figure S2. (a) Digital photographs and (b) Zeta potential of organosilica polymer sol and GO dispersed in organosilica polymer sol (GO/organosilica =1/1 wt./wt.); (c) Photographs of the blank hollow fiber (below) and the GO-Si1/1 membrane (above). The digital photographs of GO dispersed in organosilica polymer sol was taken after ultrasound. 


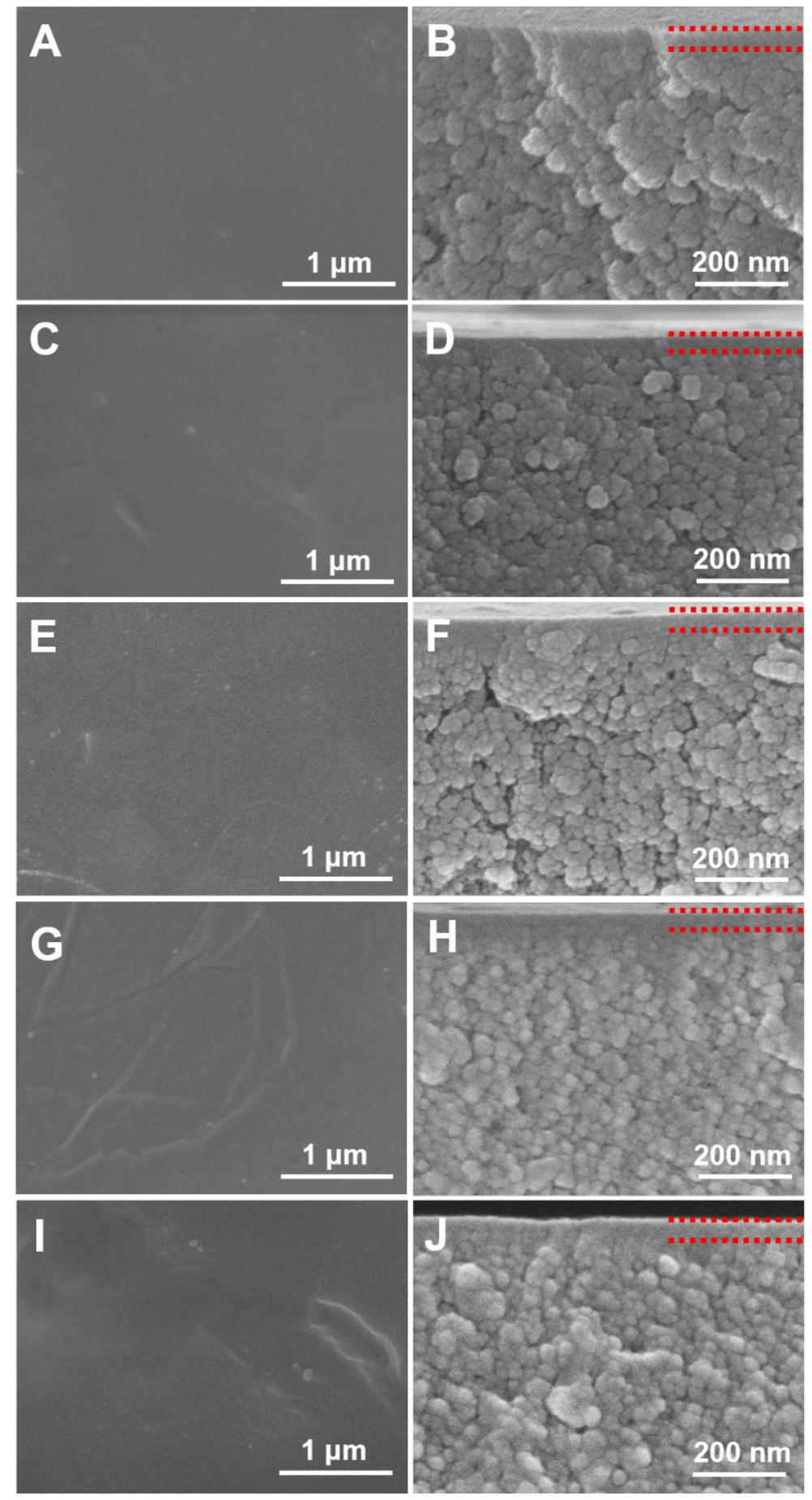

Figure S3. SEM images of the surface and cross-section view of (A, B) pure organosilica membrane, $(\mathrm{C}$, D) GO-Si1/2, (E, F) GO-Si1/1.5, (G, H) GO-Si1.5/1 and (I, J) GO-Si2/1 membranes. 


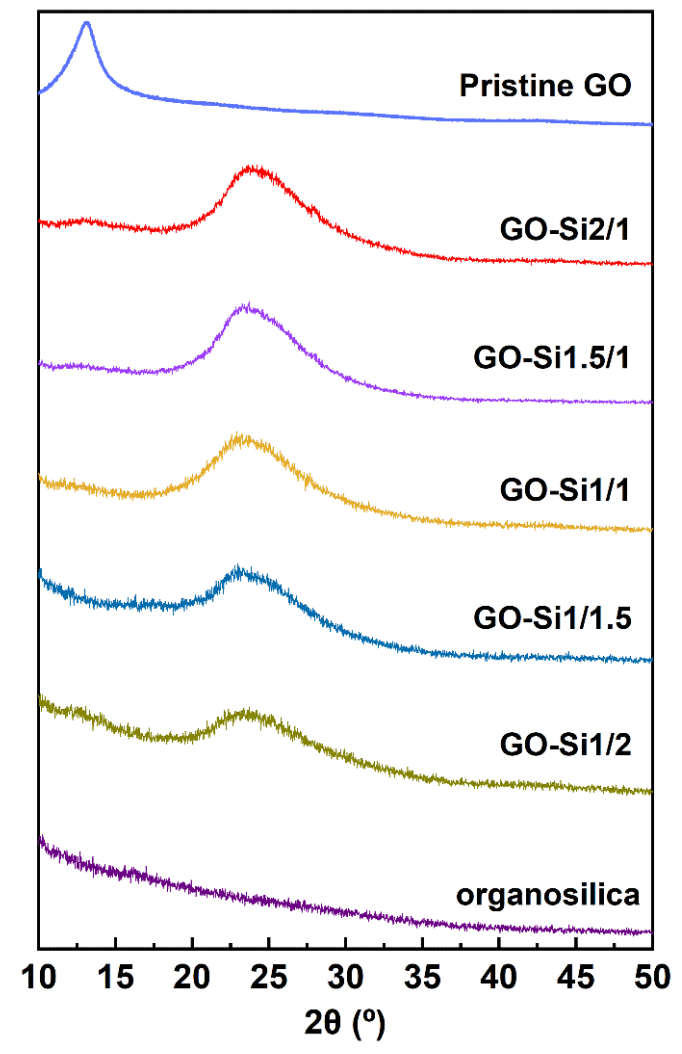

Figure S4. XRD patterns of pristine organosilica, various r-GO/organosilica membranes.

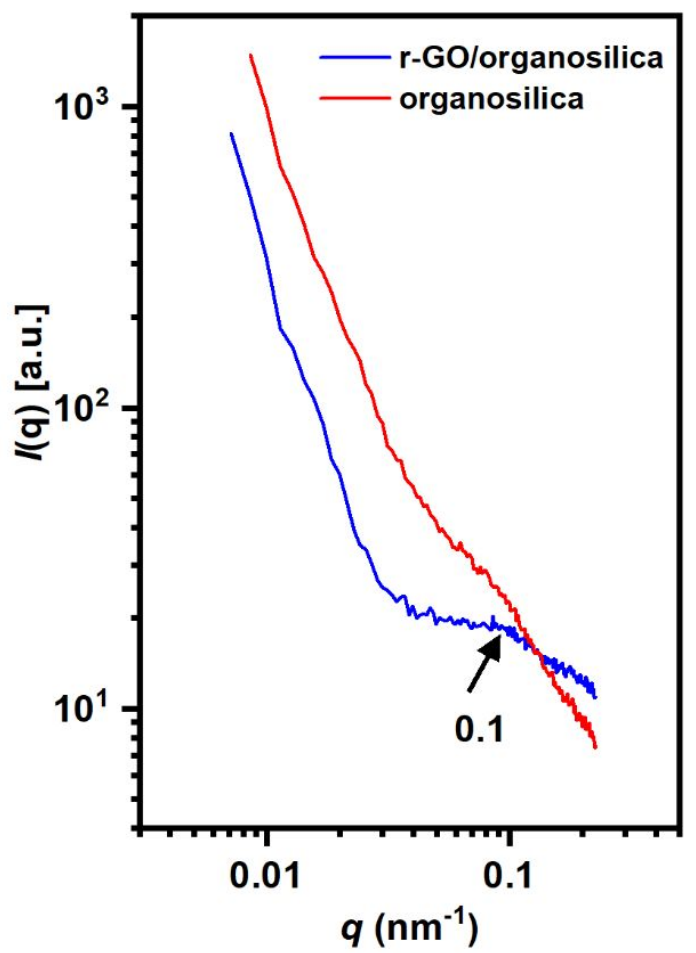

Figure S5. SAXS curves for organosilica and r-GO/organosilica, in which the X-ray beam was perpendicular to the film plane. The slope at low $q$-range (Guinier region) were used to determine fractal dimensions. 

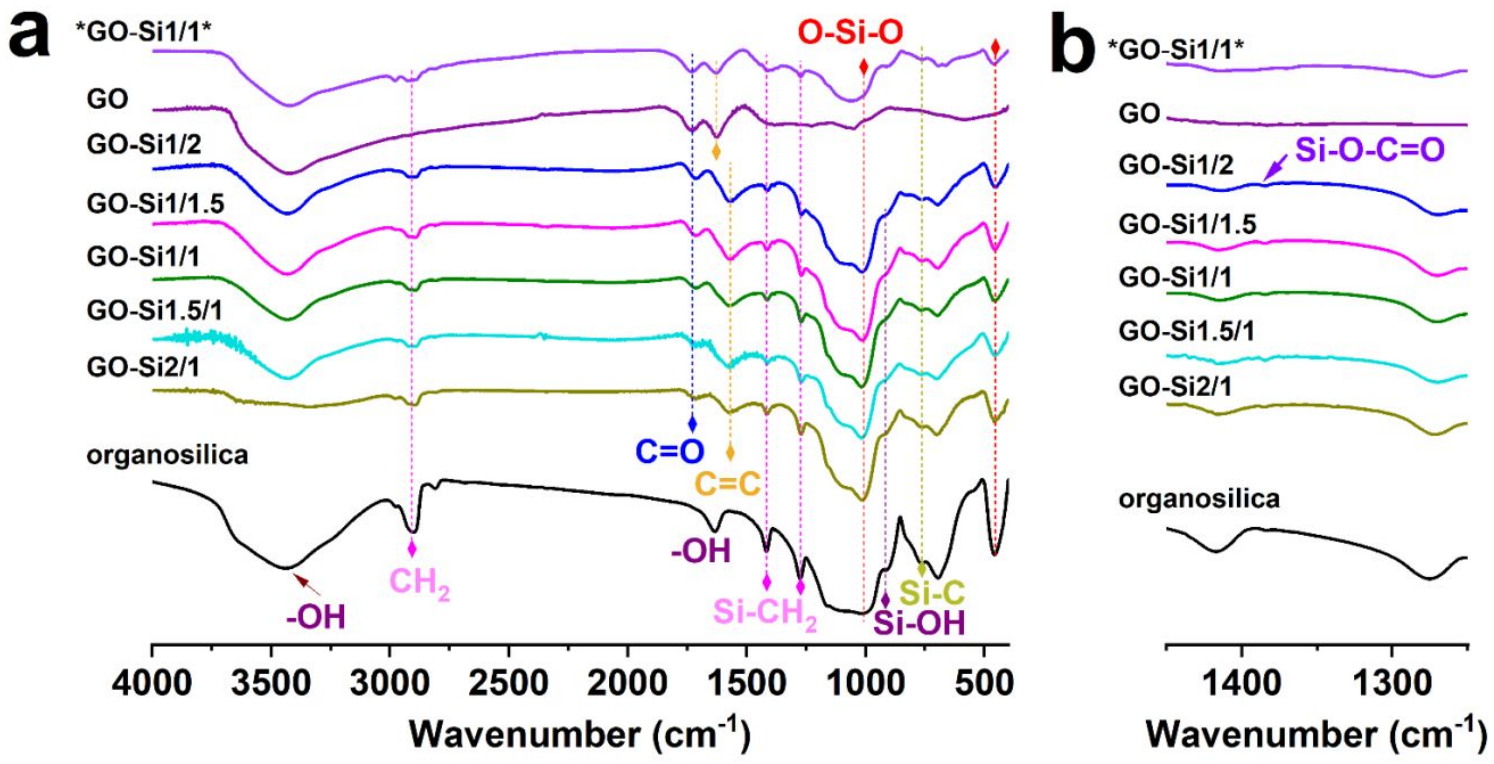

Figure S6. FT-IR spectra of GO, organosilica and r-GO/organosilica membranes, (a) from 4000 to 400 $\mathrm{cm}^{-1}$, (b) from 1450 to $1250 \mathrm{~cm}^{-1}$. ${ }^{*} \mathrm{GO}-\mathrm{Si} 1 / 1 *$ is the GO-Si1/1 membrane before $300{ }^{\circ} \mathrm{C}$ polymerization process. 


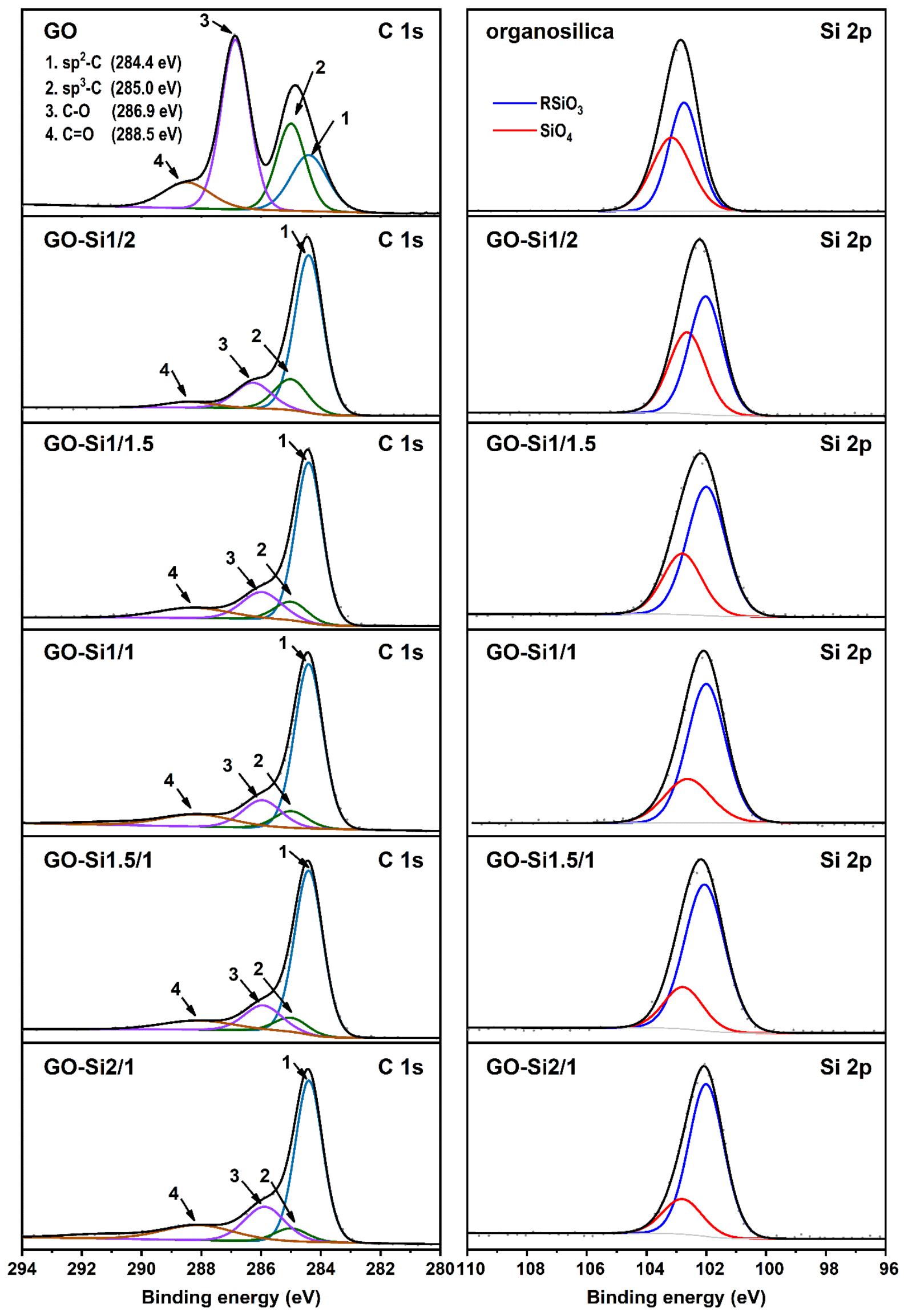

Figure S7. Evolution of C1s XPS spectrum of GO, Si 2p XPS spectrum of organosilica, and C1s and Si $2 \mathrm{p}$ XPS spectra of $\mathrm{r}-\mathrm{GO} /$ organosilica membranes. 

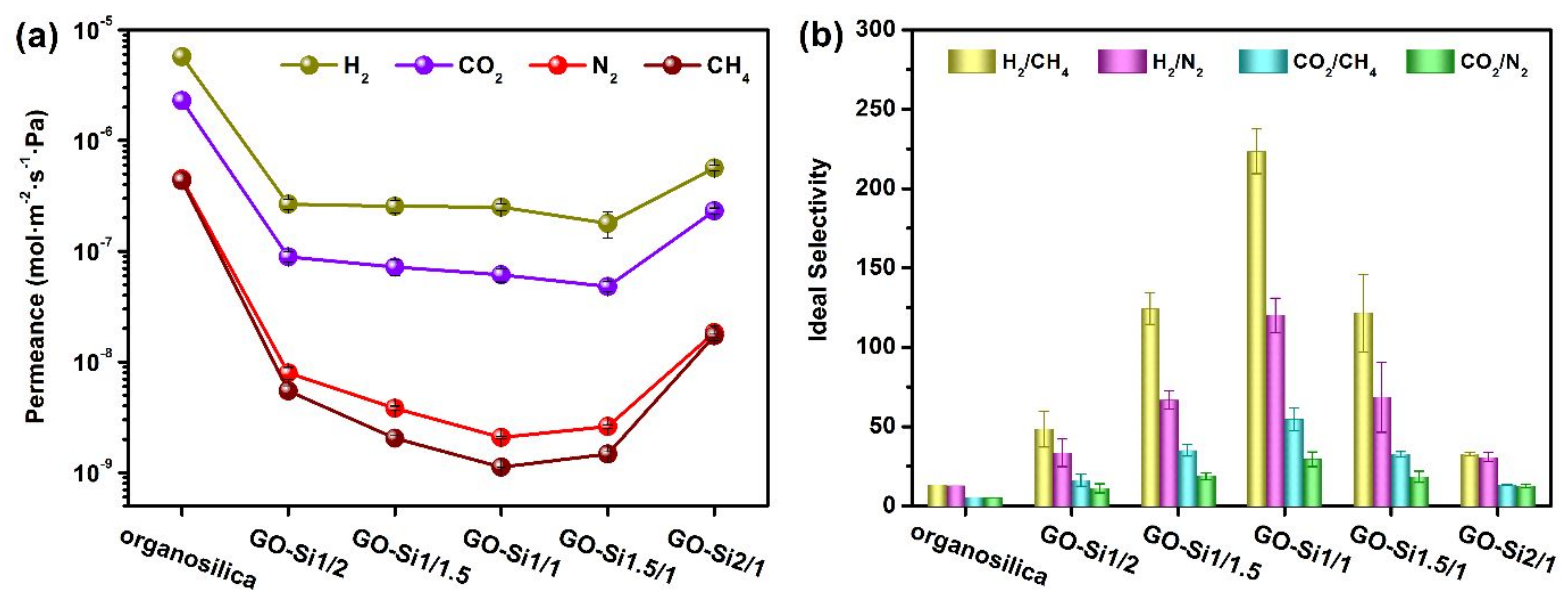

Figure S8. (a) Gas permeance and (b) the ideal selectivities for the corresponding gas pairs of the BTESEderived organosilica membrane, GO-Si1/2, GO-Si1/1.5, GO-Si1/1, GO-Si1.5/1 and GO-Si2/1 membranes. Permeances were measured at $\Delta \mathrm{p}=0.2 \mathrm{MPa}$ and $\mathrm{T}=25^{\circ} \mathrm{C}$.

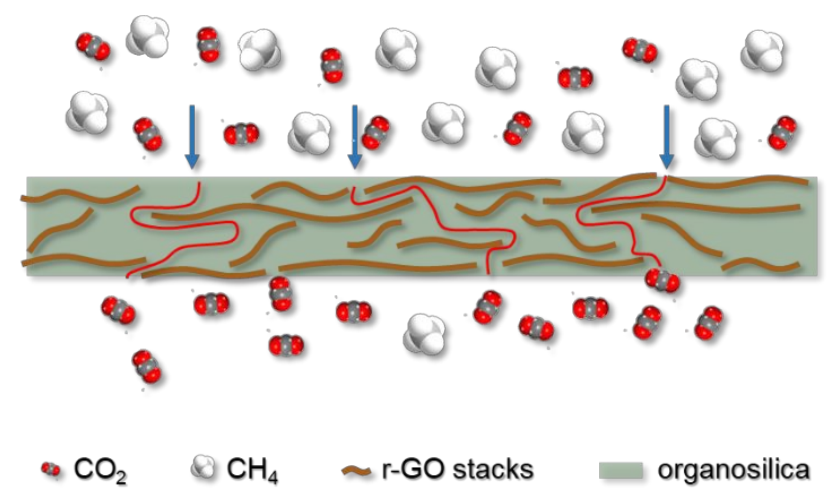

Figure S9. Schematic process of gas molecules permeating through the r-GO/organosilica membrane. 


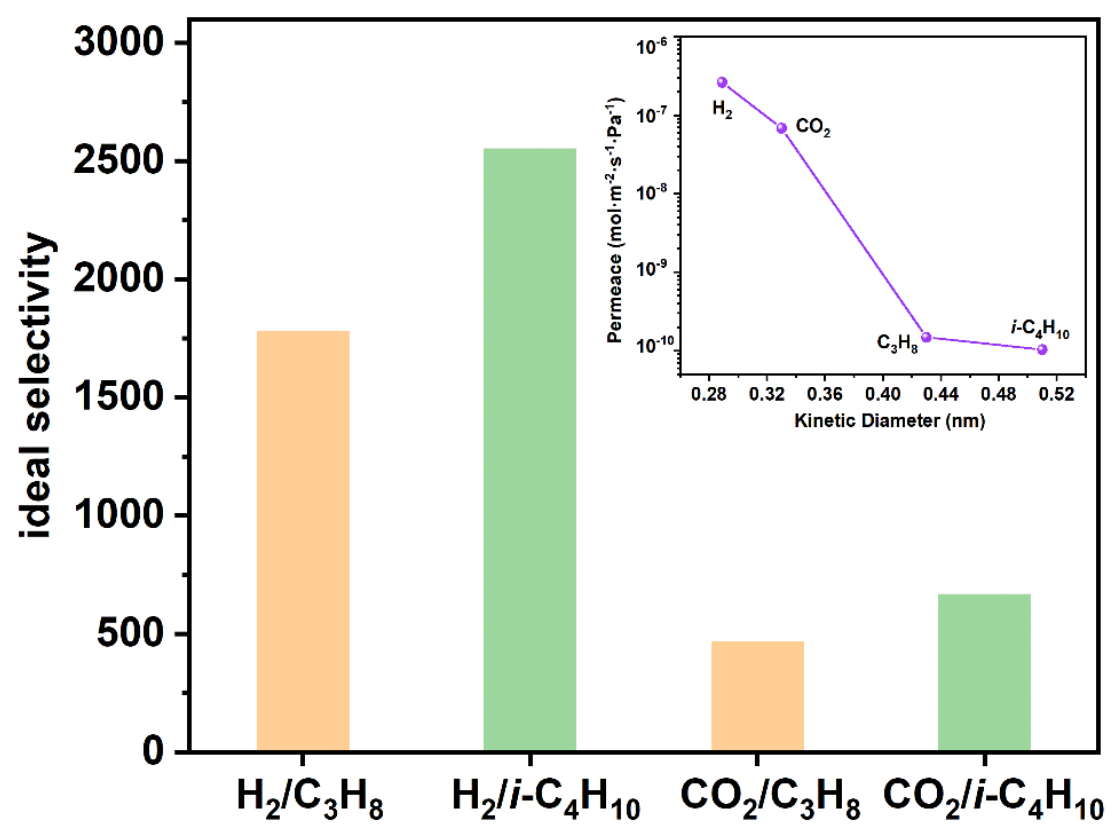

Figure S10. The ideal selectivities for $\mathrm{H}_{2} / \mathrm{C}_{3} \mathrm{H}_{8}, \mathrm{H}_{2} / i-\mathrm{C}_{4} \mathrm{H}_{10}, \mathrm{CO}_{2} / \mathrm{C}_{3} \mathrm{H}_{8}$ and $\mathrm{CO}_{2} / i-\mathrm{C}_{4} \mathrm{H}_{10}$ of GO-Si1/1 hybrid membranes (insert: single gas permeances of $\mathrm{H}_{2}, \mathrm{CO}_{2}, \mathrm{C}_{3} \mathrm{H}_{8}$ and $i-\mathrm{C}_{4} \mathrm{H}_{10}$ ). Permeance was measured at $\Delta \mathrm{p}=0.2 \mathrm{MPa}$ and $\mathrm{T}=25^{\circ} \mathrm{C}$.

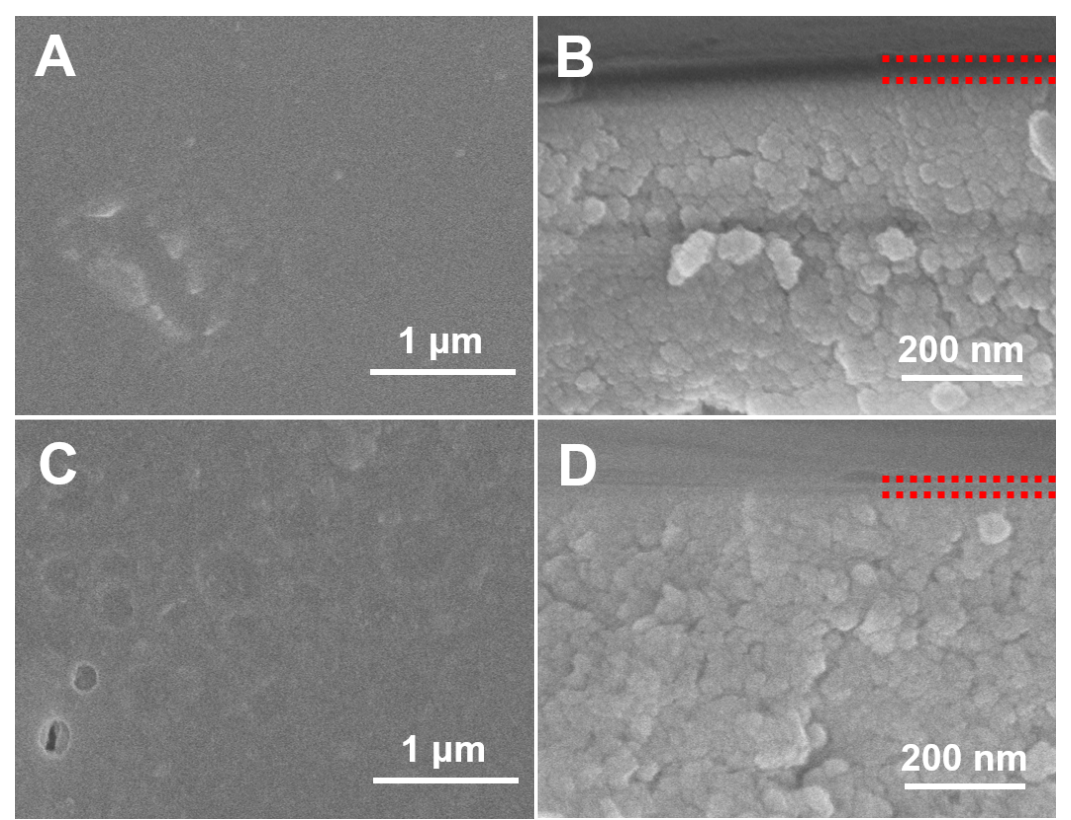

Figure S11. SEM images of the surface and cross-section view of r-GO/organosilica membranes fabricated by (A, B) 2-fold diluted coating solution and (C, D) 4-fold diluted coating solution. 

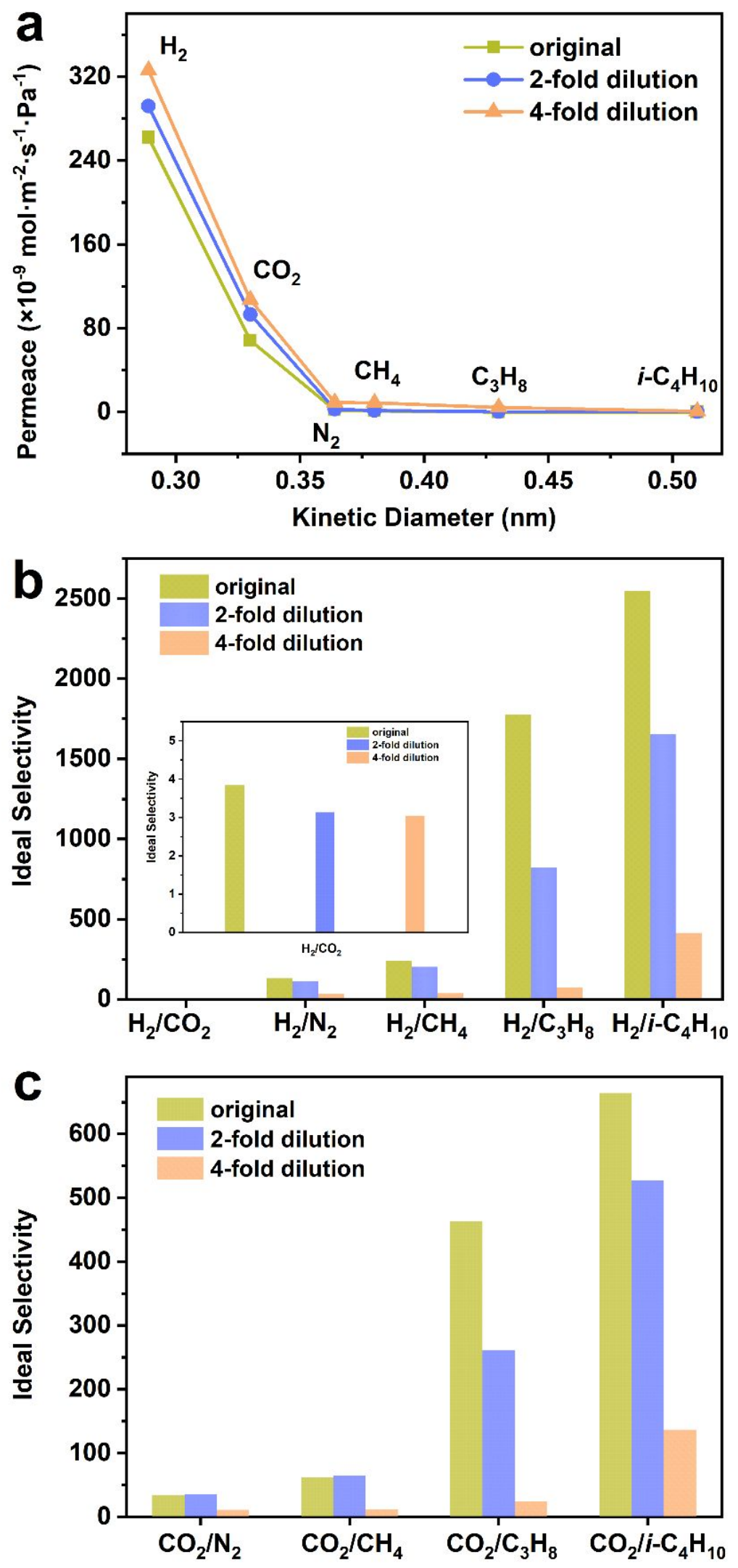

Figure S12. (a) Single gas permeances through r-GO/organosilica membranes fabricated by original, 2fold diluted and 4-fold diluted coating solution; (b-c) the ideal selectivities for the corresponding gas pairs. Permeances were measured at $\Delta \mathrm{p}=0.2 \mathrm{MPa}$ and $\mathrm{T}=25^{\circ} \mathrm{C}$. 


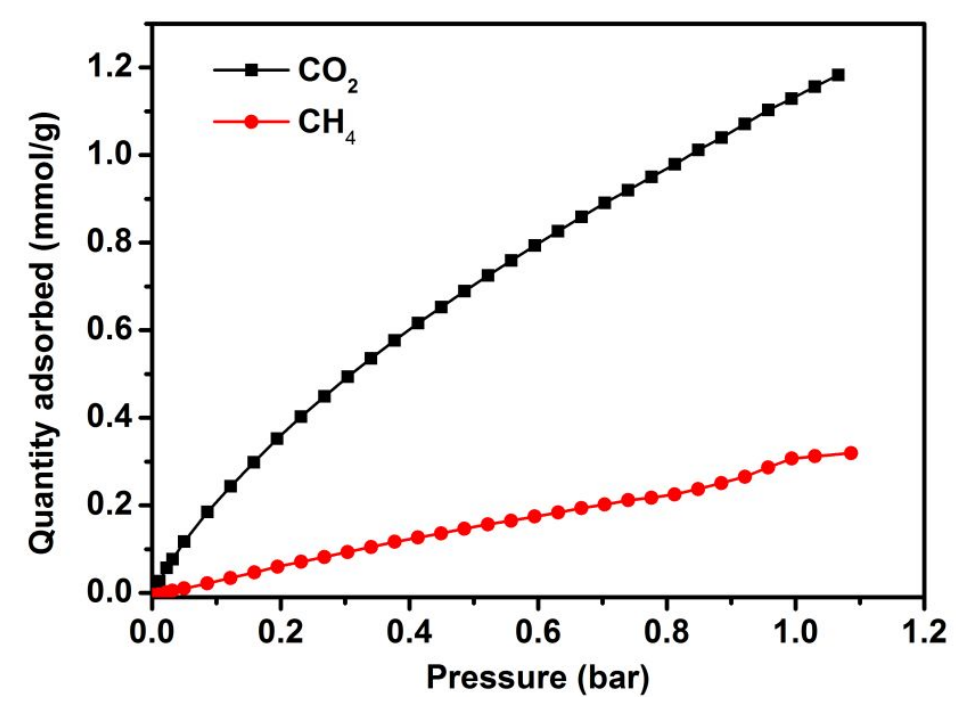

Figure S13. Pure-component $\mathrm{CO}_{2}$ and $\mathrm{CH}_{4}$ adsorptions of r-GO/organoslica powders at $25^{\circ} \mathrm{C}$.

Note: r-GO/organoslica powders show no adsorption towards single gas $\mathrm{N}_{2}$ both at $25^{\circ} \mathrm{C}$ and $150^{\circ} \mathrm{C}$, and no adsorption to $\mathrm{CO}_{2}$ and $\mathrm{CH}_{4}$ at $150^{\circ} \mathrm{C}$, so the corresponding adsorption isotherms cannot be outlined.

Calculation of $\mathrm{CO}_{2}: \mathrm{CH}_{4}$ adsorption selectivity at $25^{\circ} \mathrm{C}$ :

The adsorption selectivity of $\mathrm{CO}_{2}: \mathrm{CH}_{4}$ at $25^{\circ} \mathrm{C}$ was calculated by using the ratio of the initial slope of the gas uptake (Henry's constant) for both $\mathrm{CO}_{2}$ and $\mathrm{CH}_{4}$. Noted that the onsets of $\mathrm{CO}_{2}$ and $\mathrm{CH}_{4}$ adsorptions truly occur at the pressure nearby 0.01 bar, therefore the data points between 0.01 bar to 0.2 bar were chosen to calculate the Henry's constant (KD) shown in Figure S13.

$$
\text { AdsorptionSelectivity, } \mathrm{CO}_{2} / \mathrm{CH}_{4}=\mathrm{KD}_{\mathrm{CO} 2} / \mathrm{KD}_{\mathrm{CH} 4} \approx 5.4
$$

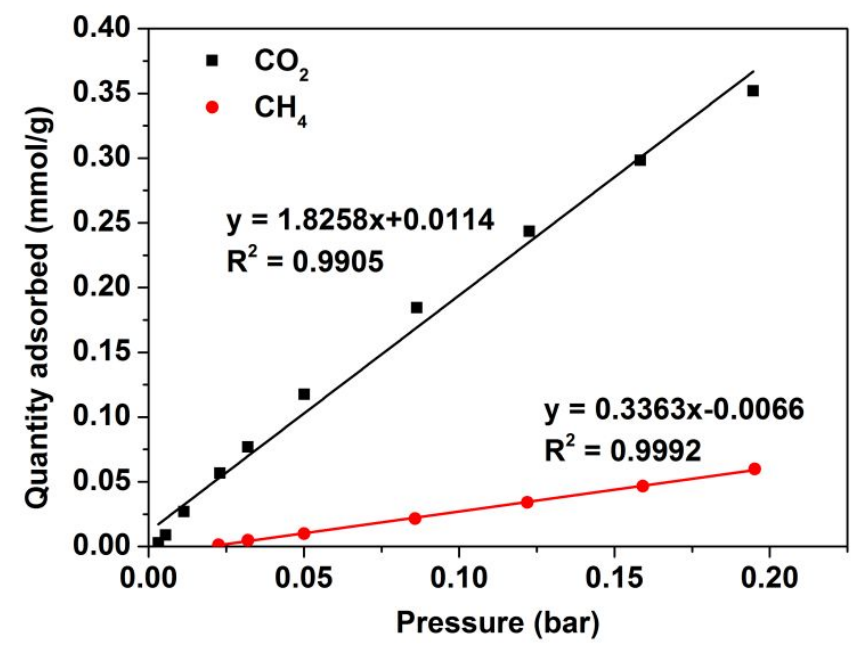

Figure S14. $\mathrm{CO}_{2}$ and $\mathrm{CH}_{4}$ adsorptions of $\mathrm{r}-\mathrm{GO}$ /organoslica at $25^{\circ} \mathrm{C}$ between 0.01 bar to 0.2 bar. 
Isosteric heat of adsorption:

The data of pure-component isotherm for $\mathrm{CO}_{2}$ and $\mathrm{CH}_{4}$ in the $\mathrm{r}-\mathrm{GO}$ /organosilica or neat organosilica samples were fitted with the single-site Langmuir model, as shown in Figure S15.

The isosteric heat of adsorption $\left(\mathrm{Q}_{\mathrm{st}}\right)$ reflects the the binding energy of the gas $\mathrm{CO}_{2}$ and $\mathrm{CH}_{4}$. The $\mathrm{Q}_{\mathrm{st}}$ for $\mathrm{CO}_{2}$ and $\mathrm{CH}_{4}$ of the r-GO/organosilica or neat organosilica samples in this study were estimated using pure-component isotherms collected at 273 and $298 \mathrm{~K}$, and calculated with the Clausius-Clapeyron equation:

$$
Q_{s t}=\frac{R T_{1} T_{2}}{T_{2}-T_{1}} \ln \frac{p_{2}}{p_{1}}
$$

where $R$ is the universal gas constant; $T_{1}$ and $T_{2}$ is the temperature 273 and $298 \mathrm{~K} ; p_{1}$ and $p_{2}$ is the pressure at 273 and $298 \mathrm{~K}$, respectively.
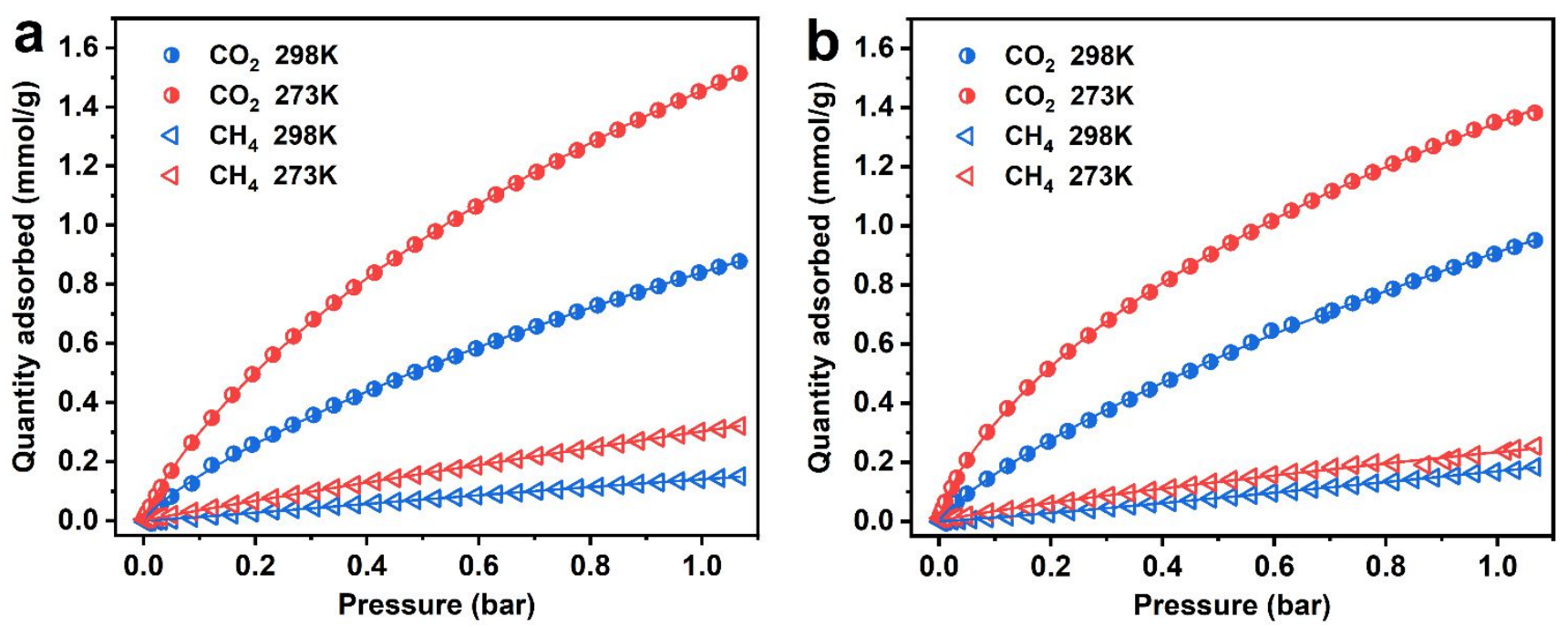

Figure S15. Sorption isotherms of neat organosilica powders (a) and r-GO organosilica powders (b) for $\mathrm{CO}_{2}$ and $\mathrm{CH}_{4}$ at $273 \mathrm{~K}$ and $298 \mathrm{~K}$. 


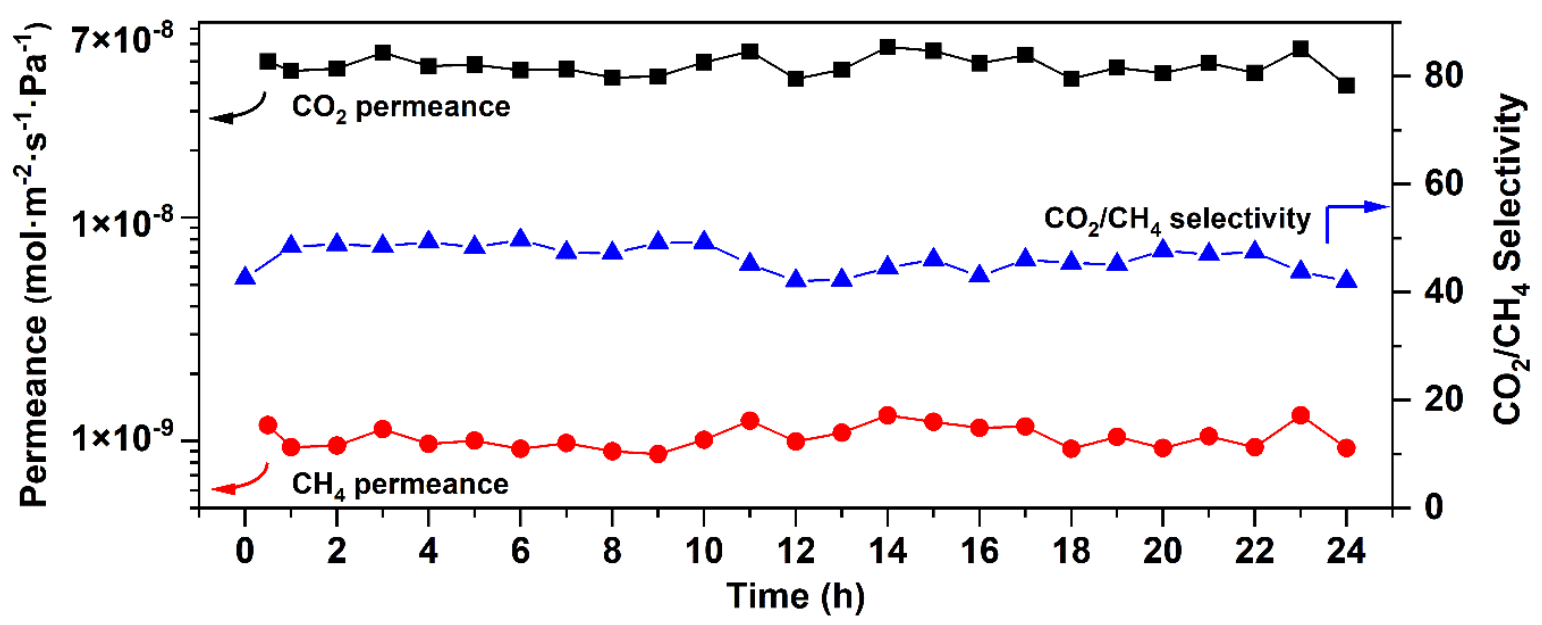

Figure S16. Thermal stability of the r-GO/organosilica membrane for the separation of an equimolar $\mathrm{CO}_{2} / \mathrm{CH}_{4}$ mixture at $25{ }^{\circ} \mathrm{C}$.

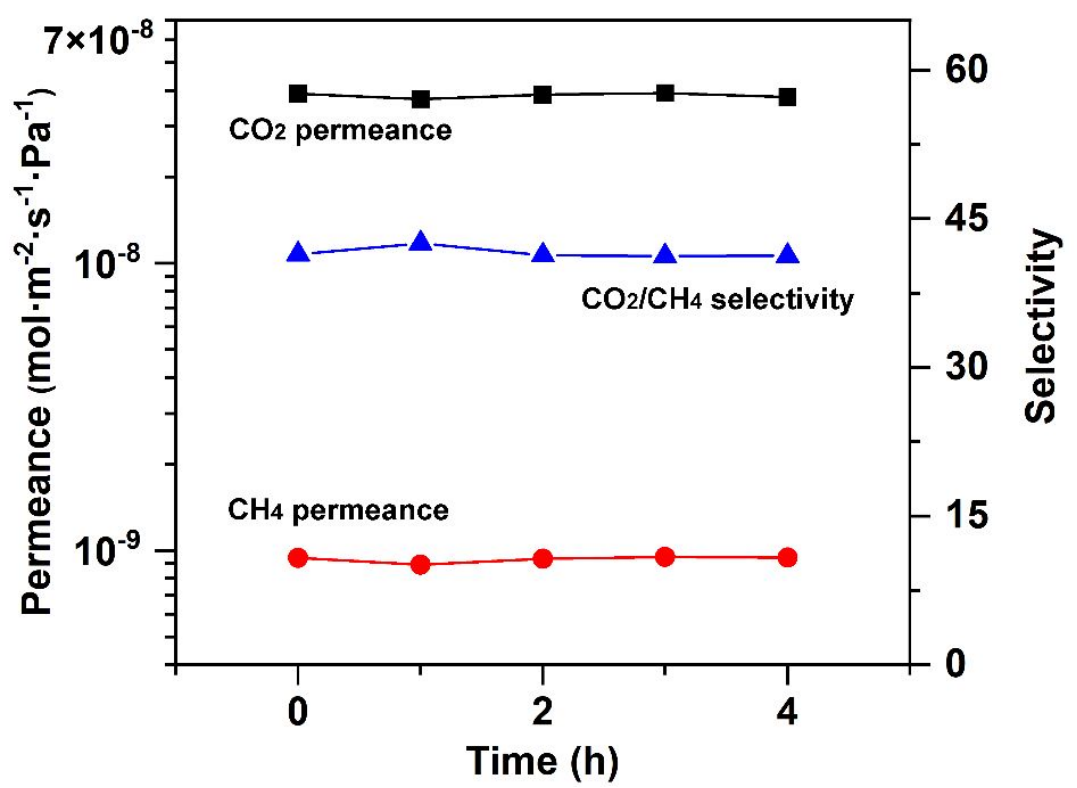

Figure S17. Thermal stability of the r-GO/organosilica membrane for the separation of an equimolar $\mathrm{CO}_{2} / \mathrm{CH}_{4}$ mixture under wet state at $150^{\circ} \mathrm{C}$. 

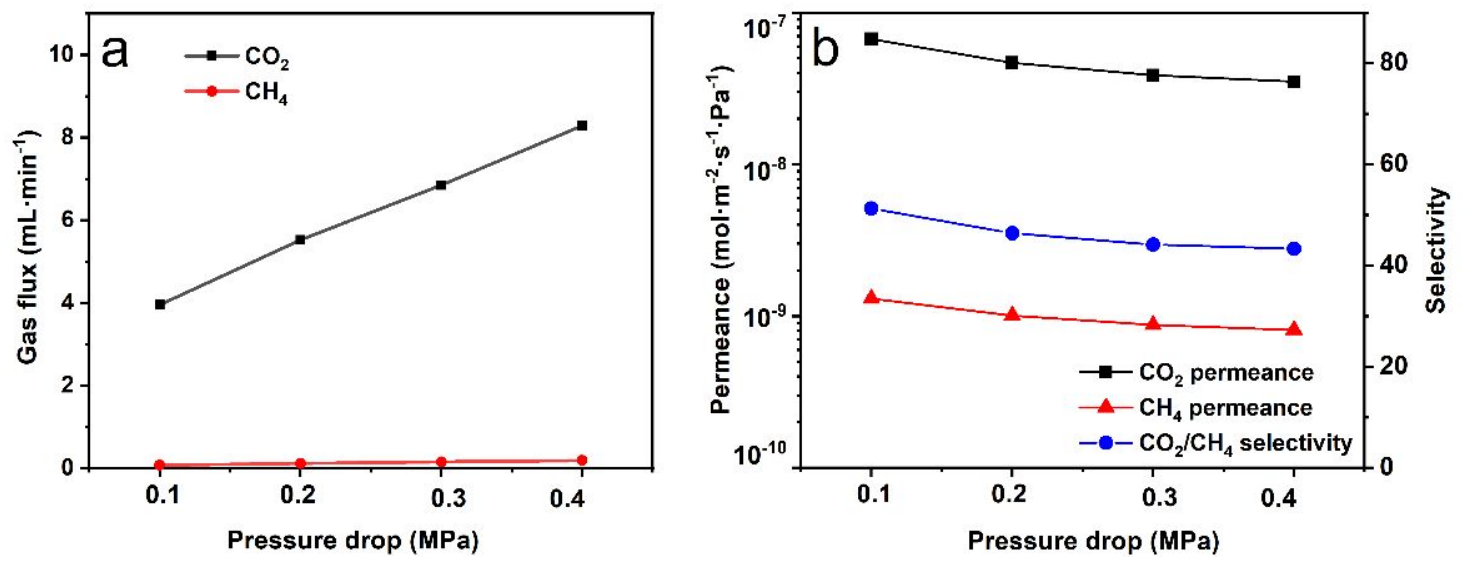

Figure S18. (a) The gas fluxes of $\mathrm{CO}_{2}$ and $\mathrm{CH}_{4}$ from an equimolar $\mathrm{CO}_{2} / \mathrm{CH}_{4}$ mixture, and (b) their gas permeances and $\mathrm{CO}_{2} / \mathrm{CH}_{4}$ selectivity of the $\mathrm{r}-\mathrm{GO} /$ organosilica membrane as function of the $\mathrm{CO}_{2}$ feed pressure at $25^{\circ} \mathrm{C}$.

Table S1. Single gas permeances of $\mathrm{H}_{2}, \mathrm{CO}_{2}$ and $\mathrm{CH}_{4}$ and the ideal selectivities of $\mathrm{H}_{2} / \mathrm{CH}_{4}$ and $\mathrm{CO}_{2} / \mathrm{CH}_{4}$ at room temperature and $0.2 \mathrm{MPa}$ of 3 tested hybrid membranes $(\mathrm{r}-\mathrm{GO} /$ organosilica $=1 / 1)$ prepared with the same procedures.

\begin{tabular}{llllll}
\hline membranes & $\begin{array}{l}\mathrm{H}_{2} \text { Permeance } \\
\left(\mathrm{mol} \cdot \mathrm{m}^{-2} \cdot \mathrm{s}^{-1} \cdot \mathrm{Pa}^{-1}\right)\end{array}$ & $\begin{array}{l}\mathrm{CO}_{2} \text { Permeance } \\
\left(\mathrm{mol} \cdot \mathrm{m}^{-2} \cdot \mathrm{s}^{-1} \cdot \mathrm{Pa}^{-1}\right)\end{array}$ & $\begin{array}{l}\mathrm{CH}_{4} \text { Permeance } \\
\left(\mathrm{mol} \cdot \mathrm{m}^{-2} \cdot \mathrm{s}^{-1} \cdot \mathrm{Pa}^{-1}\right)\end{array}$ & $\begin{array}{l}\text { Ideal Selectivity } \\
\mathrm{H}_{2} / \mathrm{CH}_{4}\end{array}$ & $\mathrm{CO}_{2} / \mathrm{CH}_{4}$ \\
\hline 1 & $2.34 \times 10^{-7}$ & $6.10 \times 10^{-8}$ & $1.11 \times 10^{-9}$ & 210.81 & 54.95 \\
2 & $2.32 \times 10^{-7}$ & $5.26 \times 10^{-8}$ & $0.986 \times 10^{-9}$ & 235.29 & 53.35 \\
3 & $2.33 \times 10^{-7}$ & $6.29 \times 10^{-8}$ & $1.11 \times 10^{-9}$ & 209.91 & 56.67 \\
\hline
\end{tabular}


Table S2. Comparison of gas separation performance of r-GO/organosilica membrane with other reported $\mathrm{H}_{2}$-selective $\mathrm{GO}$ membranes at room temperature.

\begin{tabular}{|c|c|c|c|c|}
\hline \multirow{2}{*}{ Membrane } & \multirow{2}{*}{$\begin{array}{l}\mathrm{H}_{2} \text { Permeance } \\
\left(10^{-8} \mathrm{~mol}^{\prime} \cdot \mathrm{m}^{-2} \cdot \mathrm{s}^{-1} \cdot \mathrm{Pa}^{-1}\right)\end{array}$} & \multicolumn{2}{|c|}{$\mathrm{H}_{2} / \mathrm{CH}_{4}$ Selectivity } & \multirow{2}{*}{ Ref. } \\
\hline & & Single gas & Gas Mixture & \\
\hline ZIF-8/GO & 5.46 & 1 & 11.2 & 1 \\
\hline ZIF-8/GO & 3.7 & l & 335 & 2 \\
\hline ZIF-8/rGO & 60 & 90.7 & 80.9 & 3 \\
\hline ED-GO@PSSHF & 3580 & 14.7 & / & 4 \\
\hline $\mathrm{Zn}_{2}(\mathrm{bIm})_{4}-\mathrm{GO}$ & 14 & 256 & 221 & 5 \\
\hline ZIF-8@GO & $12.7-13.5$ & 198.3 & 139.1 & 6 \\
\hline BTESE & 63.3 & 25 & 1 & 7 \\
\hline BTESE & 110 & 7.1 & / & 8 \\
\hline BTESE & 23.8 & 400 & / & 9 \\
\hline APTES-ZIF-90 & 2.9 & 1 & 71 & 10 \\
\hline TRIES & 60 & 24 & / & 11 \\
\hline SiOC membrane & $\begin{array}{l}53 \\
30 \\
24\end{array}$ & $\begin{array}{l}18 \\
50 \\
76\end{array}$ & / & 12 \\
\hline TRIES-VTMS & 100 & 15 & l & 13 \\
\hline BTESM & 100 & 150 & l & 14 \\
\hline Pd-BTESE & 23 & 31 & l & 15 \\
\hline BTESE & 27 & 24 & l & 16 \\
\hline BTESE & 37.5 & 87 & l & 17 \\
\hline Pd-BTESE & 72.6 & 15 & I & 17 \\
\hline Zr-BTESE & 7.84 & 83.6 & l & 18 \\
\hline \multirow{2}{*}{ triethoxysilane } & 23.4 & 590 & / & \multirow{2}{*}{19} \\
\hline & 54.0 & 36 & I & \\
\hline Zr-BTESE-0.20 & 18 & 400 & l & 20 \\
\hline Pd-BTESE & 21.1 & 173 & I & 21 \\
\hline BTESE & 100 & 100 & / & 22 \\
\hline ZIF-8/organosilica & 106.4 & 35 & 26.5 & 23 \\
\hline Uio-66/Matrimid & 0.26 & 1 & 80 & 24 \\
\hline $\begin{array}{l}\text { CoBDC-ns } \\
\text { /6FDA-Durene- } \\
\text { DABA }\end{array}$ & 0.172 & 41.5 & - & 25 \\
\hline R-TR-PBOIa-2h & 191 & 71 & I & 26 \\
\hline PES/MWCNTs & 2.34 & l & 44.1 & 27 \\
\hline LTA-type $\mathrm{AlPO}_{4}$ & $21-25$ & I & 4.3 & 28 \\
\hline $\mathrm{Ni}_{2}(\mathrm{~L}-\mathrm{asp})_{2}(\mathrm{bpe})$ & 100 & - & 7.7 & 29 \\
\hline r-GO/organosilica & 23 & 223.42 & 101.7 & $\begin{array}{l}\text { This } \\
\text { work }\end{array}$ \\
\hline
\end{tabular}


Table S3. Comparison of gas separation performance of r-GO/organosilica membrane with other reported $\mathrm{CO}_{2}$-selective membranes.

\begin{tabular}{|c|c|c|c|c|}
\hline \multirow{2}{*}{ Membrane } & \multirow{2}{*}{$\begin{array}{l}\mathrm{CO}_{2} \text { Permeance } \\
\left(10^{-8} \mathrm{~mol} \cdot \mathrm{m}^{-2} \cdot \mathrm{s}^{-1} \cdot \mathrm{Pa}^{-1}\right)\end{array}$} & \multicolumn{2}{|c|}{$\mathrm{CO}_{2} / \mathrm{CH}_{4}$ Selectivity } & \multirow{2}{*}{ Ref. } \\
\hline & & Single gas & Gas Mixture & \\
\hline GO-PEGDA500/PES & 5.95 & 69.5 & 66.8 & 30 \\
\hline GO/PES & 3.73 & I & 28 & 31 \\
\hline ED-GO@PSSHF & 8.5 & 0.6 & / & 32 \\
\hline GO-TFN & 3.13 & / & 25 & 33 \\
\hline Pebax/GO-DA-Zn ${ }^{2+}$ & 0.0577 & I & 30.6 & 34 \\
\hline$[\mathrm{EMIM}][\mathrm{Ac}] \mathrm{GO}-0.5$ & 1.25 & I & 39 & 35 \\
\hline Pebax-PEG-PI-GO & 0.81 & l & 45 & 36 \\
\hline rGO-PBOI & 59.76 & 32.4 & 35.1 & 37 \\
\hline rGO-ZIF-8-PEBAX & $\sim 325$ & 35 & l & 38 \\
\hline ZIF-8@GO & 0.65 & / & 8.9 & 6 \\
\hline UiO-66-GO & 0.0141 & / & 51 & 39 \\
\hline rGO/ZIF-8 & / & / & 38.45 & 40 \\
\hline $\mathrm{Zn}_{2}(\mathrm{bIm})_{4}-\mathrm{GO}$ & 14 & 2.5 & 2.4 & 5 \\
\hline ZIF-8/GO & 3.41 & / & 7.1 & 1 \\
\hline BTESE & 40.52 & 16 & 1 & 7 \\
\hline BTESE & 5.6 & 100 & / & 9 \\
\hline SPAO-34/BTESE & 24 & / & 158 & 41 \\
\hline AP-PECVD silica & 19 & 166 & / & 42 \\
\hline BTESE & 5.12 & 11.9 & l & 17 \\
\hline Pd-BTESE & 16.9 & 3.49 & l & 17 \\
\hline Zr-BTESE-0.20 & 18 & 400 & l & 20 \\
\hline Pd-BTESE & 2.37 & 11.5 & / & 21 \\
\hline BTESE & 130 & 31.5 & l & 43 \\
\hline ВTPP & 3.37 & 25 & l & 44 \\
\hline ВТРР & $\sim 10$ & 25 & / & 45 \\
\hline BTESE & 6.32 & 33 & / & 46 \\
\hline $\begin{array}{l}\text { MIL-53--NH } / \\
\text { organosilica }\end{array}$ & 14.4 & 23.2 & 18.2 & 23 \\
\hline ZIF-67/PIM & 2.18 & 16.8 & 14.9 & 47 \\
\hline $\begin{array}{l}\text { HKUST-IL/6FDA- } \\
\text { Durene }\end{array}$ & 0.61 & 29.3 & / & 48 \\
\hline $\begin{array}{l}\mathrm{Ni}_{2}(\mathrm{dobdc}) / \\
\text { 6FDA-DAM }\end{array}$ & 1.14 & 16 & 15 & 49 \\
\hline $\begin{array}{l}\text { MIL-53-NH } / \\
\text { 6FDA-DAM }\end{array}$ & 0.737 & I & 28 & 50 \\
\hline $\begin{array}{l}\text { CoBDC-ns/6FDA- } \\
\text { Durene-DABA }\end{array}$ & 0.167 & 33.6 & 23 & 25 \\
\hline GO/organosilica & 5.3 & 54.67 & 47.5 & $\begin{array}{l}\text { This } \\
\text { work }\end{array}$ \\
\hline
\end{tabular}




\section{References}

(1) Hu, Y.; Wei, J.; Liang, Y.; Zhang, H.; Zhang, X.; Shen, W.; Wang, H., Zeolitic imidazolate framework/graphene oxide hybrid nanosheets as seeds for the growth of ultrathin molecular sieving membranes. Angew. Chem. Int. Ed. 2016, 55 (6), 2048-2052.

(2) Wang, X.; Chi, C.; Tao, J.; Peng, Y.; Ying, S.; Qian, Y.; Dong, J.; Hu, Z.; Gu, Y.; Zhao, D., Improving the hydrogen selectivity of graphene oxide membranes by reducing non-selective pores with intergrown ZIF-8 crystals. Chem. Commun. 2016, 52 (52), 8087-8090.

(3) Li, W. B.; Shi, J. L.; Li, Z. J.; Wu, W. F.; Xia, Y.; Yu, Y.; Zhang, G. L., Hydrothermally reduced graphene oxide interfaces for synthesizing high-performance metal-organic framework hollow fiber membranes. Adv. Mater. Interfaces 2018, 5 (14), 7.

(4) Qi, B.; He, X.; Zeng, G.; Pan, Y.; Li, G.; Liu, G.; Zhang, Y.; Chen, W.; Sun, Y., Strict molecular sieving over electrodeposited 2D-interspacing-narrowed graphene oxide membranes. Nat. Commun. 2017, 8,825 .

(5) Li, Y.; Liu, H.; Wang, H.; Qiu, J.; Zhang, X., GO-guided direct growth of highly oriented metal organic framework nanosheet membranes for $\mathrm{H}_{2} / \mathrm{CO}_{2}$ separation. Chemical Science 2018, 9 (17), 41324141.

(6) Huang, A.; Liu, Q.; Wang, N.; Zhu, Y.; Caro, J., Bicontinuous zeolitic imidazolate framework ZIF8@GO membrane with enhanced hydrogen selectivity. J. Am. Chem. Soc. 2014, 136 (42), 14686-14689.

(7) Castricum, H. L.; Paradis, G. G.; Mittelmeijer-Hazeleger, M. C.; Bras, W.; Eeckhaut, G.; Vente, J. F.; Rothenberg, G.; ten Elshof, J. E., Tuning the nanopore structure and separation behavior of hybrid organosilica membranes. Microporous Mesoporous Mater. 2014, 185, 224-234.

(8) Kreiter, R.; Rietkerk, M. D. A.; Castricum, H. L.; van Veen, H. M.; ten Elshof, J. E.; Vente, J. F., Evaluation of hybrid silica sols for stable microporous membranes using high-throughput screening. $J$. Sol-Gel Sci. Technol. 2011, 57 (3), 245-252.

(9) Castricum, H. L.; Qureshi, H. F.; Nijmeijer, A.; Winnubst, L., Hybrid silica membranes with enhanced hydrogen and $\mathrm{CO}_{2}$ separation properties. J. Membr. Sci. 2015, 488, 121-128.

(10) Huang, A.; Wang, N.; Kong, C.; Caro, J., Organosilica-Functionalized Zeolitic Imidazolate Framework ZIF-90 Membrane with High Gas-Separation Performance. Angew. Chem. Int. Ed. 2012, 51 (42), 10551-10555.

(11) Kanezashi, M.; Matsugasako, R.; Tawarayama, H.; Nagasawa, H.; Yoshioka, T.; Tsuru, T., Tuning the pore sizes of novel silica membranes for improved gas permeation properties via an in situ reaction between NH3 and Si-H groups. Chem. Commun. 2015, 51 (13), 2551-2554.

(12) Kanezashi, M.; Sazaki, H.; Nagasawa, H.; Yoshioka, T.; Tsuru, T., Preparation and gas permeation properties of thermally stable organosilica membranes derived by hydrosilylation. J. Mater. Chem. A 2014, 2 (3), 672-680.

(13) Kanezashi, M.; Sazaki, H.; Nagasawa, H.; Yoshioka, T.; Tsuru, T., Evaluating the gas permeation properties and hydrothermal stability of organosilica membranes under different hydrosilylation conditions. J. Membr. Sci. 2015, 493, 664-672.

(14) Kanezashi, M.; Yoneda, Y.; Nagasawa, H.; Tsuru, T.; Yamamoto, K.; Ohshita, J., Gas permeation properties for organosilica membranes with different $\mathrm{Si} / \mathrm{C}$ ratios and evaluation of microporous structures. AlChE J. 2017, 63 (10), 4491-4498.

(15) Lei, J.; Song, H.; Wei, Y.; Zhao, S.; Qi, H., A novel strategy to enhance hydrothermal stability of Pd-doped organosilica membrane for hydrogen separation. Microporous Mesoporous Mater. 2017, 253, 55-63.

(16) Qureshi, H. F.; Nijmeijer, A.; Winnubst, L., Influence of sol-gel process parameters on the microstructure and performance of hybrid silica membranes. J. Membr. Sci. 2013, 446, 19-25.

(17) Song, H.; Zhao, S.; Lei, J.; Wang, C.; Qi, H., Pd-doped organosilica membrane with enhanced gas permeability and hydrothermal stability for gas separation. J. Mater. Sci. 2016, 51 (13), 6275-6286.

(18) Song, H.; Zhao, S.; Chen, J.; Qi, H., Hydrothermally stable Zr-doped organosilica membranes for $\mathrm{H}_{2} / \mathrm{CO}_{2}$ separation. Microporous Mesoporous Mater. 2016, 224, 277-284. 
(19) Tanaka, T.; Kanezashi, M.; Nagasawa, H.; Tsuru, T., Effects of Calcination Condition on the Network Structure of Triethoxysilane (TRIES) and How Si-H Groups Influence Hydrophobicity Under Hydrothermal Conditions. Ind. Eng. Chem. Res. 2019, 58 (9), 3867-3875.

(20) ten Hove, M.; Nijmeijer, A.; Winnubst, L., Facile synthesis of zirconia doped hybrid organic inorganic silica membranes. Sep. Purif. Technol. 2015, 147, 372-378.

(21) Wei, Y.; Zhang, H.; Lei, J.; Song, H.; Qi, H., Controlling pore structures of Pd-doped organosilica membranes by calcination atmosphere for gas separation. Chin. J. Chem. Eng. 2019, 27 (12), 3036-3042. (22) Yu, X.; Nagasawa, H.; Kanezashi, M.; Tsuru, T., Improved thermal and oxidation stability of bis( triethoxysilyl) ethane ( BTESE)- derived membranes, and their gas-permeation properties. J. Mater. Chem. A 2018, 6 (46), 23378-23387.

(23) Kong, C.; Du, H.; Chen, L.; Chen, B., Nanoscale MOF/organosilica membranes on tubular ceramic substrates for highly selective gas separation. Energy Environ. Sci. 2017, 10 (8), 1812-1819.

(24) Friebe, S.; Geppert, B.; Steinbach, F.; Caro, J., Metal-organic framework UiO-66 layer: A highly oriented membrane with good selectivity and hydrogen permeance. ACS Appl. Mater. Interfaces 2017, 9 (14), 12878-12885.

(25) Bi, X.; Zhang, Y. a.; Zhang, F.; Zhang, S.; Wang, Z.; Jin, J., MOF nanosheet-based mixed matrix membranes with metal-organic coordination interfacial interaction for gas separation. ACS Appl. Mater. Interfaces 2020, DOI: 10.1021/acsami.0c14639.

(26) Zhuang, Y.; Seong, J. G.; Lee, W. H.; Do, Y. S.; Lee, M. J.; Wang, G.; Guiver, M. D.; Lee, Y. M., Thermally rearranged (TR) random/block poly(benzoxazole-co-imide) gas separation membranes. Macromolecules 2015, 48 (15), 5286-5299.

(27) Ghomshani, A. D.; Ghaee, A.; Mansourpour, Z.; Esmaili, M.; Sadatnia, B., Improvement of $\mathrm{H}_{2} / \mathrm{CH}_{4}$ separation performance of PES hollow fiber membranes by addition of MWCNTs into polymeric matrix. Polym.-Plast. Technol. Eng. 2016, 55 (11), 1155-1166.

(28) Huang, A.; Liang, F.; Steinbach, F.; Gesing, T. M.; Caro, J., Neutral and cation-free LTA-type aluminophosphate $\left(\mathrm{ALPO}_{4}\right)$ molecular sieve membrane with high hydrogen permselectivity. J. Am. Chem. Soc. 2010, 132 (7), 2140-2141.

(29) Kang, Z.; Fan, L.; Wang, S.; Sun, D.; Xue, M.; Qiu, S., In situ confinement of free linkers within a stable MOF membrane for highly improved gas separation properties. CrystEngComm 2017, 19 (12), 1601-1606.

(30) Wang, S.; Xie, Y.; He, G.; Xin, Q.; Zhang, J.; Yang, L.; Li, Y.; Wu, H.; Zhang, Y.; Guiver, M. D.; Jiang, Z., Graphene oxide membranes with heterogeneous nanodomains for efficient $\mathrm{CO}_{2}$ separations. Angew. Chem. Int. Ed. 2017, 56 (45), 14246-14251.

(31) Kim, H. W.; Yoon, H. W.; Yoon, S.-M.; Yoo, B. M.; Ahn, B. K.; Cho, Y. H.; Shin, H. J.; Yang, H.; Paik, U.; Kwon, S.; Choi, J.-Y.; Park, H. B., Selective gas transport through few-layered graphene and graphene oxide membranes. Science 2013, 342 (6154), 91-95.

(32) Qi, B.; He, X.; Zeng, G.; Pan, Y.; Li, G.; Liu, G.; Zhang, Y.; Chen, W.; Sun, Y., Strict molecular sieving over electrodeposited 2D-interspacing-narrowed graphene oxide membranes. Nat. Commun. 2017, 8.

(33) Wong, K. C.; Goh, P. S.; Ismail, A. F., Highly permeable and selective graphene oxide-enabled thin film nanocomposite for carbon dioxide separation. Int. J. Greenh. Gas Con. 2017, 64, 257-266.

(34) Peng, D.; Wang, S.; Tian, Z.; Wu, X.; Wu, Y.; Wu, H.; Xin, Q.; Chen, J.; Cao, X.; Jiang, Z., Facilitated transport membranes by incorporating graphene nanosheets with high zinc ion loading for enhanced $\mathrm{CO}_{2}$ separation. J. Membr. Sci. 2017, 522, 351-362.

(35) Karunakaran, M.; Villalobos, L. F.; Kumar, M.; Shevate, R.; Akhtar, F. H.; Peinemann, K. V., Graphene oxide doped ionic liquid ultrathin composite membranes for efficient $\mathrm{CO}_{2}$ capture. J. Mater. Chem. A 2017, 5 (2), 649-656.

(36) Li, X.; Cheng, Y.; Zhang, H.; Wang, S.; Jiang, Z.; Guo, R.; Wu, H., Efficient $\mathrm{CO}_{2}$ capture by functionalized graphene oxide nanosheets as fillers to fabricate multi-permselective mixed matrix membranes. ACS Appl. Mater. Interfaces 2015, 7 (9), 5528-5537. 
(37) Kim, S.; Hou, J.; Wang, Y.; Ou, R.; Simon, G. P.; Seong, J. G.; Lee, Y. M.; Wang, H., Highly permeable thermally rearranged polymer composite membranes with a graphene oxide scaffold for gas separation. J. Mater. Chem. A 2018, 6 (17), 7668-7674.

(38) Jamil, N.; Othman, N. H.; Alias, N. H.; Shahruddin, M. Z.; Roslan, R. A.; Lau, W. J.; Ismail, A. F., Mixed matrix membranes incorporated with reduced graphene oxide (rGO) and zeolitic imidazole framework-8 (ZIF-8) nanofillers for gas separation. J. Solid State Chem. 2019, 270, 419-427.

(39) Castarlenas, S.; Tellez, C.; Coronas, J., Gas separation with mixed matrix membranes obtained from MOF UiO-66-graphite oxide hybrids. J. Membr. Sci. 2017, 526, 205-211.

(40) Jamil, N.; Othman, N. H.; Shahrudin, M. Z.; Razlan, M. R. M.; Alias, N. H.; Marpani, F.; Jye, L. W.; Goh, P. S.; Ismail, A. F., EFFECTS OF PEBAX COATING CONCENTRATIONS ON $\mathrm{CO}_{2} / \mathrm{CH}_{4}$ SEPARATION OF RGO/ZIF-8 PES MEMBRANES. Jurnal Teknologi 2020, 82 (2), 51-60.

(41) Mu, Y.; Chen, H.; Xiang, H.; Lan, L.; Shao, Y.; Fan, X.; Hardacre, C., Defects-healing of SAPO34 membrane by post-synthesis modification using organosilica for selective CO2 separation. J. Membr. Sci. 2019, 575, 80-88.

(42) Nagasawa, H.; Yamamoto, Y.; Tsuda, N.; Kanezashi, M.; Yoshioka, T.; Tsuru, T., Atmosphericpressure plasma-enhanced chemical vapor deposition of microporous silica membranes for gas separation. J. Membr. Sci. 2017, 524, 644-651.

(43) Yang, X.; Du, H.; Lin, Y.; Song, L.; Zhang, Y.; Gao, X.; Kong, C.; Chen, L., Hybrid organosilica membrane with high $\mathrm{CO} 2$ permselectivity fabricated by a two-step hot coating method. J. Membr. Sci. 2016, 506, 31-37.

(44) Yu, L.; Kanezashi, M.; Nagasawa, H.; Ohshita, J.; Naka, A.; Tsuru, T., Fabrication and microstructure tuning of a pyrimidine-bridged organoalkoxysilane membrane for $\mathrm{CO}_{2}$ separation. Ind. Eng. Chem. Res. 2017, 56 (5), 1316-1326.

(45) Yu, L.; Kanezashi, M.; Nagasawa, H.; Oshita, J.; Naka, A.; Tsuru, T., Pyrimidine-bridged organoalkoxysilane membrane for high-efficiency $\mathrm{CO}_{2}$ transport via mild affinity. Sep. Purif. Technol. 2017, 178, 232-241.

(46) Li, J.-Y.; Wang, D. K.; Tseng, H.-H.; Wey, M.-Y., Solvent effects on diffusion channel construction of organosilica membrane with excellent $\mathrm{CO}_{2}$ separation properties. J. Membr. Sci. 2021, 618, 118758 .

(47) Wu, X.; Liu, W.; Wu, H.; Zong, X.; Yang, L.; Wu, Y.; Ren, Y.; Shi, C.; Wang, S.; Jiang, Z., Nanoporous ZIF-67 embedded polymers of intrinsic microporosity membranes with enhanced gas separation performance. J. Membr. Sci. 2018, 548, 309-318.

(48) Ling, R.; Ge, L.; Diao, H.; Rudolph, V.; Zhu, Z., Ionic Liquids as the MOFs/Polymer Interfacial Binder for Efficient Membrane Separation. ACS Appl. Mater. Interfaces 2016, 8 (46), 32041-32049.

(49) Bachman, J. E.; Smith, Z. P.; Li, T.; Xu, T.; Long, J. R., Enhanced ethylene separation and plasticization resistance in polymer membranes incorporating metal-organic framework nanocrystals. Nat. Mater. 2016, 15 (8), 845-849.

(50) Sabetghadam, A.; Seoane, B.; Keskin, D.; Duim, N.; Rodenas, T.; Shahid, S.; Sorribas, S.; Le Guillouzer, C.; Clet, G.; Tellez, C.; Daturi, M.; Coronas, J.; Kapteijn, F.; Gascon, J., Metal organic framework crystals in mixed-matrix membranes: impact of the filler morphology on the gas separation performance. Adv. Funct. Mater. 2016, 26 (18), 3154-3163. 